\title{
Optimal power flow under uncertainty: An extensive out-of-sample analysis
}

Arrigo, Adriano; Ordoudis, Christos; Kazempour, Jalal; De Grève, Zacharie; Toubeau, Jean-François; Vallée, François

\section{Published in:}

Proceedings of 2019 IEEE PES Innovative Smart Grid Technologies Europe

Link to article, DOI:

10.1109/isgteurope.2019.8905752

Publication date:

2019

Document Version

Peer reviewed version

Link back to DTU Orbit

Citation (APA):

Arrigo, A., Ordoudis, C., Kazempour, J., De Grève, Z., Toubeau, J-F., \& Vallée, F. (2019). Optimal power flow under uncertainty: An extensive out-of-sample analysis. In Proceedings of 2019 IEEE PES Innovative Smart Grid Technologies Europe IEEE. https://doi.org/10.1109/isgteurope.2019.8905752

\section{General rights}

Copyright and moral rights for the publications made accessible in the public portal are retained by the authors and/or other copyright owners and it is a condition of accessing publications that users recognise and abide by the legal requirements associated with these rights.

- Users may download and print one copy of any publication from the public portal for the purpose of private study or research.

- You may not further distribute the material or use it for any profit-making activity or commercial gain

- You may freely distribute the URL identifying the publication in the public portal 


\title{
Optimal Power Flow Under Uncertainty: An Extensive Out-of-Sample Analysis
}

\author{
Adriano Arrigo $^{1}$, Christos Ordoudis ${ }^{2}$, Jalal Kazempour ${ }^{2}$, Zacharie De Grève ${ }^{1}$, \\ Jean-François Toubeau ${ }^{1}$ and François Vallée ${ }^{1}$ \\ ${ }^{1}$ Electrical Power Engineering Unit, University of Mons, Mons, Belgium \\ \{adriano.arrigo, zacharie.degreve, jean-francois.toubeau, francois.vallee\}@umons.ac.be \\ ${ }^{2}$ Department of Electrical Engineering, Technical University of Denmark, Kgs. Lyngby, Denmark \\ \{chror, seykaz\}@elektro.dtu.dk
}

\begin{abstract}
The uncertainty induced by high penetration of stochastic generation in power systems requires to be properly taken into account within Optimal Power Flow (OPF) problems to make informed day-ahead decisions that minimize the social cost in view of potential balancing actions. This ends up in a two-stage OPF problem that is usually solved using two-stage stochastic programming or adaptive robust optimization. Another alternative is the use of chance-constrained programming that allows to control the conservativeness of the decisions. In this paper, we aim at defining a fair basis for assessing the performance of these three techniques, using an extensive out-of-sample evaluation. Considering a common wind power database, each technique leads to optimal day-ahead decisions that are a posteriori assessed through the real-time stage on unseen realizations of the uncertainty. Our main conclusion is that undertaking conservative decisions results in lower standard deviations of the cost, but at the expense of higher expected cost.
\end{abstract}

Index Terms-Stochastic programming, adaptive robust optimization, chance-constrained programming, optimal power flow, out-of-sample analysis.

\section{NOMENCLATURE}

Sets

$g \in \mathcal{G}_{(n)}$

$l \in \mathcal{L}_{(n)}$

$q \in \mathcal{Q}_{(n)}$

$\omega \in \Omega$

$n \in \mathcal{N}$

$m \in \mathcal{M}_{n}$

$\mathrm{x}$

$\mathbf{y}$

$C_{g}$

$C_{g}^{\mathrm{U} / \mathrm{D}}$

$R_{g}^{\mathrm{U} / \mathrm{D}, \max }$

$P_{g}^{\max }$

$P_{l}$

$V_{l}^{\text {curt }}$

$P_{q}$

$\Delta P_{q}^{+/- \text {max }}$

$F_{m n}^{\max }$

$F_{m n}$

$\pi_{\omega}$

$\Delta P_{q}$

$\Delta P_{q}^{+/-}$
$P_{g}$
$\delta_{n}^{0}$
$r_{g}^{\mathrm{U} / \mathrm{D}}$
$P_{q}^{\text {spill }}$
$P_{l}^{\text {curt }}$
$\delta_{n}$

$\Delta P_{q}^{+/-}$

$P_{g}$

$\delta_{n}^{0}$

$r_{g}^{\mathrm{U} / \mathrm{D}}$

$P_{q}^{\text {spill }}$

$\delta_{n}$
Wind power excess/deficit of farm $q$ in the real-time stage [MW].

Day-ahead decision variables

Dispatch of generator $g$ [MW].

Scheduled voltage angle at bus $n$ [rad].

Real-time decision variables

Upward/downward regulation of generator $g$ [MW].

Wind power spillage of farm $q$ [MW].

Curtailed load of load $l$ [MW].

Real-time voltage angle at bus $n$ [rad].

\section{INTRODUCTION}

Intergovernmental agreements and electricity sector liberalization promoted the deployment of renewable energy sources in power systems, thereby increasing the share of uncertain and intermittent power production in the whole generation mix. In a liberalized environment, the aim for the system operator is to maximize the social welfare by cost-effectively scheduling the generators while ensuring the balance between generation and consumption, supply-side and demand-side restrictions and grid limits. This problem is referred to as the Optimal Power Flow (OPF) problem. With significant wind penetration, the day-ahead (DA) stage decisions of the OPF problems need to become more uncertainty-aware. One solution, which is still aligned with the current practice, is to preserve the deterministic description of uncertainties and impose minimum reserve requirements. The other solution is to make DA stage energy-only and non-deterministic, i.e., the DA stage takes into account the forecast distribution (rather than a point forecast) and the plausible actions at the realtime (RT) stage. The latter is the focus of this paper. There exist several optimization techniques to model such a twostage (i.e., DA and RT) problem affected by uncertainties.

Two-stage stochastic programming is a well-known technique that minimizes the expected value of the objective function by representing the distribution of an uncertain parameter through a set of scenarios, each assigned with a probability [1]. However, the dimension of the problem increases with the number of scenarios, which hinders its practical utilization. It is therefore important to generate a limited but representative set of scenarios with assigned probabilities, which is itself a challenging task and is not straightforward for the system operators (acquisition and processing of probabilistic data). Furthermore, the resulting decisions may be risky because they 
might not be cost-efficient against extreme scenarios that are usually assigned with low probability.

To overcome those issues, adaptive robust optimization [2] gained interest over the last decade. In this technique, the distribution of the uncertainty is no longer described by scenarios but instead by a continuous uncertainty set, which is usually more straightforward to acquire and less computationally expensive. The objective function is optimized for the worst-case realization while ensuring that the undertaken decisions remain feasible over the entire uncertainty space. Consequently, decisions are usually over-conservative.

Chance-constrained programming [3] bridges the gap between stochastic programming and robust optimization. Indeed, the system operator is now able to achieve the desired risk attitude and control the conservativeness of the decisions by stating that the violation probability of uncertainty-affected constraints is kept smaller than a predefined level of confidence. Chance-constrained problems are hard to solve; however, recent developments allow for tractable reformulations and approximations [4].

Authors in [5] solve a two-stage probabilistic OPF problem using two techniques, stochastic optimization and robust optimization. They compare the results through an out-of-sample analysis, and show the benefits of robust solutions. Authors in [3] use chance constraints which are analytically reformulated assuming the distribution of the uncertainties is gaussian and, highlighting the usefulness of controlling the risk attitude. References [6] and [7] propose hybrid methods combining the strength of scenario-based and interval methods for tackling uncertainty in the unit commitment problem and compare them with stochastic programming and robust optimization.

In this paper, we aim at comparing the aforementioned uncertainty modeling techniques in terms of resulting system cost. To address this comparison, we solve the two-stage OPF problem, considering a common wind power database to characterize the uncertainty. Next, we perform an out-ofsample analysis, meaning that the quality of the DA decisions is evaluated through numerous unseen realizations at the RT stage. This analysis provides a fair basis for comparison because the out-of-sample data are kept the same for each implemented technique but differ from the in-sample database.

Our work distinguishes from the literature in terms of plurality of implemented techniques. Indeed, we bring together two-stage stochastic OPF, adaptive robust OPF and chanceconstrained OPF and compare them through an extensive out-of-sample analysis, without making any assumption on the distribution of the uncertain parameter. The main trend observed in the results is that conservative decisions comes with a higher expected cost but are less sensitive to the actual realization of uncertain parameters, i.e., the variance of the out-of-sample cost distribution is reduced.

The paper is organized as follows. Section II further introduces the OPF under uncertainty and its reformulations. Section III evaluates the performance of DA decisions through an out-of-sample analysis, while Section IV concludes on the main results and prospects.

\section{OPF UNDER UNCERTAINTY}

The formulation of the OPF problem in Section II-B requires some assumptions that are presented in Section II-A. The implementations associated with each optimization approach are presented in Sections II-C, II-D and II-E.

\section{A. Assumptions}

In this work, we consider two settlements including DA and RT stages, and exclude the other trading floors, e.g., intraday stages. The DA stage is solved the day before delivery considering a single period of one hour (i.e., power in MW is equal to energy in MWh), discarding all inter-temporal constraints. The RT stage is then solved at the moment of delivery which enables the available resources to compensate (if necessary) the deviations that may occur. There exist several possible RT actions: the upward or downward regulation capability allows the generators to deviate from their DA schedule by increasing or decreasing their output power, the load curtailment representing the consumption reduction for loads and the wind power spillage which corresponds to a part of wind power that cannot be injected and is spilled.

For the sake of clarity, we make some additional assumptions. Firstly, an energy-only scheme equivalent to the one presented in [1] is adopted. Secondly, the demand is supposed to be inelastic and deterministic, meaning that consumers are willing to be supplied at any price and that the load will not deviate from the forecast. In such a case, maximizing the social welfare is equivalent to minimizing the social cost. Thirdly, the wind power production and wind spillage action are considered cost-free. This is close to reality because of the free renewable energy sources and the governmental incentives. Finally, we use a lossless DC approximation for power flows.

\section{B. Deterministic Formulation}

Under the assumptions described in Section II-A, the DA stage is written as the OPF problem (1), which is a linear program:

$$
\begin{aligned}
& \min _{P_{g}, \delta_{n}^{0}} \sum_{g \in \mathcal{G}} C_{g} P_{g} \\
& \text { s.t. } P_{g}^{\min } \leq P_{g} \leq P_{g}^{\max } \forall g \in \mathcal{G} \\
& \sum_{g \in \mathcal{G}_{n}} P_{g}+\sum_{q \in \mathcal{Q}_{n}} P_{q}=\sum_{l \in \mathcal{L}_{n}} P_{l}+\sum_{m \in \mathcal{M}_{n}} B_{m n}\left(\delta_{n}^{0}-\delta_{m}^{0}\right) \quad \forall n \in \mathcal{N} \\
& B_{m n}\left(\delta_{n}^{0}-\delta_{m}^{0}\right) \leq F_{m n}^{\max } \forall n \in \mathcal{N} \forall m \in \mathcal{M}_{n} \\
& \delta_{n}^{0} \text { free } \forall n \in \mathcal{N} \backslash\left\{n_{\text {ref }}\right\}, \quad \delta_{n}^{0}=0 \quad\left(n: n_{\text {ref }}\right) .
\end{aligned}
$$

The objective function (1a) to be minimized represents the total operating cost. Constraint (1b) represents the power limits of the generators. Equation (1c) ensures that production matches demand for each node of the system. Constraint (1d) imposes a maximum capacity for each line while (1e) defines the voltage angle at each node of the network. For given DA schedules, the RT stage is solved to adjust the wind power deviations $\Delta P_{q}$ via OPF problem (2) which is also a linear program: 


$$
\begin{aligned}
& \min _{\substack{r_{g}^{\mathrm{U}}, r_{g}^{\mathrm{D}}, P_{q}^{\text {spill }} \\
P_{l}^{\text {curt }}, \delta_{n}}} \sum_{g \in \mathcal{G}}\left(C_{g}^{\mathrm{U}} r_{g}^{\mathrm{U}}-C_{g}^{\mathrm{D}} r_{g}^{\mathrm{D}}\right)+\sum_{l \in \mathcal{L}} V_{l}^{\text {curt }} P_{l}^{\text {curt }} \\
\text { s.t. } & \sum_{g \in \mathcal{G}_{n}}\left(r_{g}^{\mathrm{U}}-r_{g}^{\mathrm{D}}\right)+\sum_{q \in \mathcal{Q}_{n}}\left(\Delta P_{q}-P_{q}^{\text {spill }}\right)+\sum_{l \in \mathcal{L}_{n}} P_{l}^{\text {curt }} \\
& =\sum_{m \in \mathcal{M}_{n}} B_{m n}\left(\delta_{n}-\delta_{n}^{0}-\delta_{m}+\delta_{m}^{0}\right) \quad \forall n \in \mathcal{N} \\
& P_{g}+r_{g}^{\mathrm{U}} \leq P_{g}^{\max } \forall g \in \mathcal{G} \\
& P_{g}-r_{g}^{\mathrm{D}} \geq 0 \forall g \in \mathcal{G} \\
& 0 \leq P_{l}^{\text {curt }} \leq P_{l} \forall l \in \mathcal{L} \\
& 0 \leq P_{q}^{\text {spill }} \leq P_{q}+\Delta P_{q} \forall q \in \mathcal{Q} \\
& 0 \leq r_{g}^{\mathrm{U} / \mathrm{D}} \leq R_{g}^{\mathrm{U} / \mathrm{D}, \max } \forall g \in \mathcal{G} \\
& B_{m n}\left(\delta_{n}-\delta_{m}\right) \leq F_{m n}^{\max } \forall n \in \mathcal{N} \forall m \in \mathcal{M}_{n} \\
& \delta_{n} \text { free } \forall n \in \mathcal{N} \backslash\left\{n_{\text {ref }}\right\}, \quad \delta_{n}=0\left(n: n_{\text {ref }}\right) .
\end{aligned}
$$

The objective function (2a) corresponds to the RT operational costs (generator regulation costs and value of curtailed load). The nodal power balance in RT is ensured by (2b) while power limits of the generators are enforced by (2c) and (2d). Constraints (2e)-(2f) respectively require that the amount of curtailed load (spilled wind power) lies between zero and the real consumption (real wind power generation). Regulation limits of generators are enforced by $(2 \mathrm{~g})$. The capacity of transmission lines is imposed by $(2 \mathrm{~h})$ and the voltage angle at each node of the network is given by (2i).

In the following, we describe three ways for solving this probabilistic OPF problem. In order to write the OPF problems in a compact way, let us define $\mathbf{x}$ the DA decisions $\left\{P_{g}, \delta_{n}^{0}\right\}, \mathbf{y}$ the RT decisions $\left\{r_{g}^{\mathrm{U} / \mathrm{D}}, P_{q}^{\text {spill }}, P_{l}^{\text {curt }}, \delta_{n}\right\}, \operatorname{cost}^{\mathrm{DA}}(\mathbf{x})$ the linear function of DA operational costs (1a) and $\operatorname{cost}^{\mathrm{RT}}\left(\mathbf{x}, \mathbf{y}, \Delta P_{q}\right)$ the linear function of RT operational costs (2a).

\section{Two-Stage Stochastic OPF Formulation}

The two-stage stochastic OPF (S-OPF), whose goal is to minimize the expected value of the operational costs among given scenarios, is compactly written in model (3) as follows:

$$
\begin{aligned}
& \min _{\mathbf{x}, \mathbf{y}_{\omega}} \operatorname{cost}^{\mathrm{DA}}(\mathbf{x})+\mathbb{E}_{\omega}\left[\operatorname{cost}^{\mathrm{RT}}\left(\mathbf{x}, \mathbf{y}_{\omega}, \Delta P_{q \omega}\right)\right] \\
& \text { s.t. DA constraints (1b)-(1e) } \\
& \quad \text { RT constraints (2b)-(2i) } \forall \omega \in \Omega .
\end{aligned}
$$

The objective function (3a) consists of the DA costs and the expected value of the RT costs among scenarios $\omega \in \Omega$. Day-ahead constraints are imposed through (3b) while RT constraints for each scenario $\omega$ are enforced by (3c).

Since the stochastic solution may be too risky under extreme events, we may use the Conditional Value-at-Risk (CVaR) [8] as a risk measure. The risk-averse form of S-OPF (3) becomes then:

$$
\begin{gathered}
\min _{\mathbf{x}, \mathbf{y}_{\omega}} \begin{array}{r}
(1-k) \mathbb{E}_{\omega} \\
{\left[\operatorname{cost}^{\mathrm{DA}}(\mathbf{x})+\operatorname{cost}^{\mathrm{RT}}\left(\mathbf{x}, \mathbf{y}_{\omega}, \Delta P_{q \omega}\right)\right]} \\
\\
\quad+k \mathrm{CVaR}_{\alpha}\left(\mathbf{x}, \mathbf{y}_{\omega}, \Delta P_{q \omega}\right)
\end{array} \\
\text { s.t. Constraints (3b) and (3c) } \\
\text { CVaR-related constraints (see [8] for more details) }
\end{gathered}
$$

where objective function (4a) is a weighted sum of the expected value of the cost among all scenarios and the linear risk function $\mathrm{CVaR}_{\alpha}($.$) that consists of the expected value$ among the $100(1-\alpha) \%$ worst scenarios. The weight of risk term in (4a), can be adjusted by parameter $0 \leq k \leq 1$.

\section{Adaptive Robust OPF Formulation}

The Adaptive Robust OPF (AR-OPF) problem (5) is:

$$
\begin{gathered}
\min _{\mathbf{x}} \operatorname{cost}^{\mathrm{DA}}(\mathbf{x})+\max _{\Delta \mathbf{P}_{\mathbf{q}}} \min _{\mathbf{y}} \operatorname{cost}^{\mathrm{RT}}\left(\mathbf{x}, \mathbf{y}, \Delta P_{q}\right) \\
\text { s.t. } \mathrm{RT} \text { constraints (2b)-(2i) } \\
\text { s.t. } \Delta P_{q} \in \mathcal{U}
\end{gathered}
$$$$
\text { s.t. DA constraints (1b)-(1e). }
$$

The AR-OPF problem (5) is a min-max-min problem that cannot be solved directly. Through the max operator in objective function (5a), nature is seeking the realization $\Delta \mathbf{P}_{\mathbf{q}}$ inside the uncertainty set $\mathcal{U}$ that maximizes the costs. Reference [5] proposes a decomposition method which has been adopted in this work. The first step for solving (5) is to derive the dual optmization problem of the inner-min problem, resulting in an objective function that contains bilinear terms. Strong duality theorem allows to recover linearity, which is the major contribution of [5]. The max operators can then be merged ending up in a linear min-max problem containing complementarity conditions in the constraints. A FortunyAmat (Big-M) reformulation of these conditions makes the problem mixed-integer and linear. The resulting min-max problem is afterwards decomposed and solved through a primal cut algorithm. The main advantages of this robust method are the guaranteed convergence towards the global optimum, the possible use of any polyhedral uncertainty set and a comparatively low number of involved binary variables.

In AR-OPF (5), the worst-case deviation of wind power $\Delta P_{q}$ is determined endogenously (treated as a variable). A key element when designing robust problems is therefore, the size and shape of the uncertainty set $\mathcal{U}$ because it impacts the decisions and their conservativeness. In this paper, the latter is chosen to be polyhedral, allowing the overall program to remain linear and, is described by (6) which represents a modified version of the budget-type uncertainty set:

$$
\begin{aligned}
& \Delta P_{q}=\Delta P_{q}^{+}-\Delta P_{q}^{-} \forall q \in \mathcal{Q} \\
& 0 \leq \Delta P_{q}^{+} \leq \Delta P_{q}^{+, \text {max }} \forall q \in \mathcal{Q} \\
& 0 \leq \Delta P_{q}^{-} \leq \Delta P_{q}^{-, \max } \forall q \in \mathcal{Q} \\
& \sum_{q \in \mathcal{Q}}\left(\frac{\Delta P_{q}^{+}}{\Delta P_{q}^{+, \text {max }}}+\frac{\Delta P_{q}^{-}}{\Delta P_{q}^{-, \max }}\right) \leq \Gamma .
\end{aligned}
$$

Equation (6a) defines positive and negative wind power deviations while (6b) and (6c) limit those deviations. Equation (6d) enforces an upper bound $\Gamma$ to the sum of relative absolute deviations, meaning that the aggregation of wind farms cannot deviate extremely and simultaneously in the same direction.

\section{E. Chance-Constrained OPF Formulation}

The chance-constrained programming provides a flexibility for the system operator to control the conservativeness. The 
compact Chance-Constrained OPF (CC-OPF) is (7) given by:

$$
\begin{aligned}
& \min _{\mathbf{x}, \mathbf{y}} \operatorname{cost}^{\mathrm{DA}}(\mathbf{x})+\operatorname{cost}^{\mathrm{RT}}\left(\mathbf{x}, \mathbf{y}, \Delta P_{q}\right) \\
& \text { s.t. } \text { DA constraints }(1 \mathrm{~b})-(1 \mathrm{e}) \\
& \quad V\left(\mathbf{x}, \mathbf{y}, \Delta P_{q}\right)=\mathbb{P}(\mathrm{RT} \text { constraints }(2 \mathrm{~b})-(2 \mathrm{i})) \geq 1-\epsilon .
\end{aligned}
$$

The joint chance constraint (7c) enforces the realization probability of RT constraints $V\left(\mathbf{x}, \mathbf{y}, \Delta P_{q}\right)$ to be equal to or higher than a predefined level of confidence $1-\epsilon$, but makes the problem non-convex. A mathematical reformulation of such a constraint is not straightforward in general and necessitates the knowledge of the multivariate distribution function followed by the uncertain parameters (see [3]). In this work, we use a sampling-based approach [9] that does not require the distribution information, to reformulate the joint chance constraint (7c). To this end, we randomly pick $N$ samples from the historical data, with $N$ obtained by (8):

$$
N \geq\left\lceil\frac{1}{\epsilon} \frac{e}{e-1}\left(n_{x}-1+\ln \left(\frac{1}{\beta}\right)\right)\right\rceil
$$

where $e$ is Euler number, $n_{x}$ is the number of decision variables, $\beta$ is the level of confidence ensuring the solution to be an $\epsilon$-level feasible solution, and the operator $[1$ picks the smallest integer higher than the argument. The objective function is therefore defined as the expectation over those samples and the original CC-OPF problem recasts as a S-OPF.

Pursuing a similar idea, one can recast the original CC-OPF problem (7) as an AR-OPF by enclosing the same $N$ samples within a continuous box uncertainty set. This reformulation is used in this paper to solve CC-OPF as an AR-OPF problem.

\section{Case Study: Out-OF-S ample Analysis}

A modified version of the IEEE 24-bus Reliability Test System is used in this case study. It consists of 12 generators, 17 loads and 4 wind farms connected to a 24-bus network. The wind penetration level (total expected wind power to total load ratio) is equal to $29.6 \%$. The modified network and the related input parameters are provided in [10]. We use a wind power dataset which includes 10,000 samples representing the output power of 15 wind farms during 43 consecutive hours. We arbitrarily pick one hour (hour 37 ) and four wind farms $(1,2,3$ and 12) and rescale these data based on the installed wind capacity. We then use 6,000 out of 10,000 samples to characterize the uncertainty within the models; the remaining 4,000 samples are used later for out-of-sample analysis. Fig. 1(a) illustrates the in-sample and out-of-sample data for arbitrarily selected two (out of four) wind farms.

We first solve each two-stage OPF problem (i.e., S-OPF, AR-OPF and CC-OPF) and obtain the optimal DA decisions and system cost for each of those techniques. Then, for given DA decisions, we solve the deterministic version of OPF problem in RT stage, i.e., problem (2), for each out-of-sample realization, from which we attain the total system cost in DA and RT, amount of curtailed load and spilled wind power. We can next calculate the mean and standard deviation of those

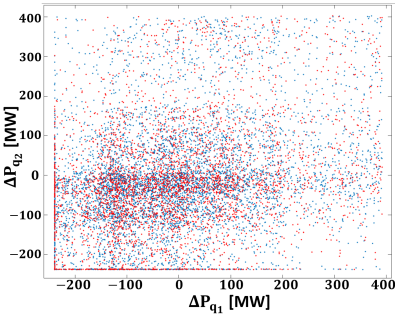

(a) In-sample (blue) and out-ofsample (red) data showing the wind power deviation in RT with respect to the DA forecast.

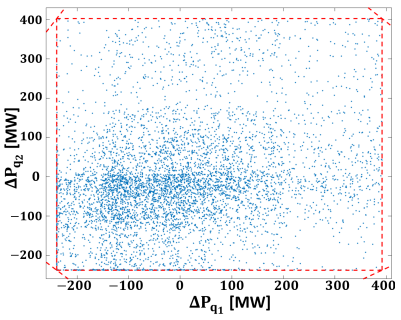

(c) Budget-type uncertainty set for AR-OPF enclosing all original 6,000 in-sample data

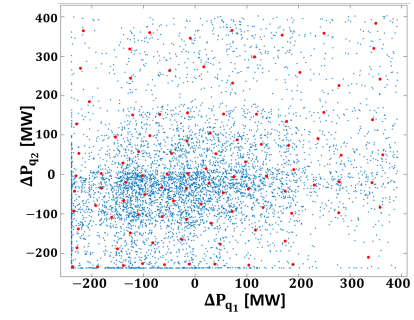

(b) The reduced set of 100 insample scenarios (red) selected based on Kantorovitch distance

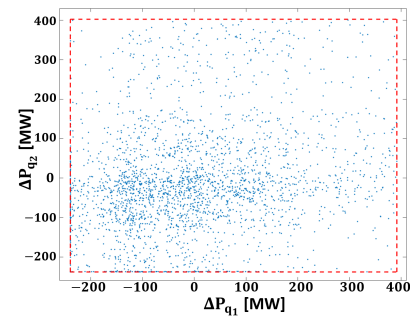

(d) Ramdomly chosen scenarios under CC-OPF and corresponding robust set for $\epsilon=0.05$
Fig. 1: Wind power data

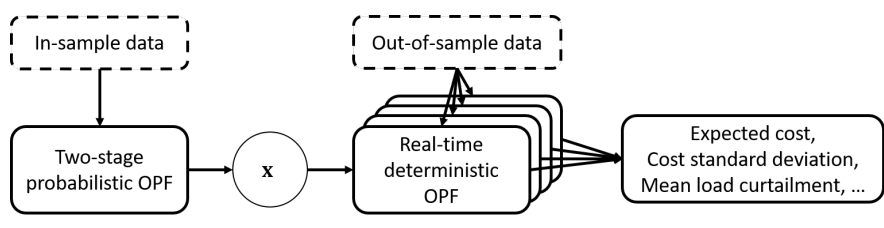

Fig. 2: The process of out-of-sample analysis

quantities. Fig. 2 summarizes how the out-of-sample analysis is performed. We now present in detail how we solved each probabilistic OPF problem.

Deterministic OPF: this problem is solved based on a single forecast, i.e., the expected in-sample wind power.

S-OPF: we use a scenario reduction algorithm based on Kantorovitch probability metric [11] to avoid computational burden. With this algorithm, 30 or 100 scenarios are selected among the original 6,000 samples in order to minimize Kantorovitch distance between the resulting set and the overall set of samples. Each selected scenario is then assigned with a probability based on the number of closest samples. The set of 100 scenarios is shown in Fig. 1(b). A risk-averse version of S-OPF is also solved considering $k=1$ (full risk-aversion) and $\alpha=0.95$ (the 5\% worst scenarios are taken into account).

AR-OPF: we generate four different uncertainty sets, three of which enclosing a given subset of in-sample dataset $(6,000$, 1,000 or 100 samples) and one enclosing the reduced set of 30 scenarios. Parameters $\Delta P_{q}^{-, \max }$ and $\Delta P_{q}^{+, \max }$ are treated as the maximum negative and positive deviations for each farm $q$, whereas $\Gamma$ in (6d) is computed as the maximum total relative deviation among those scenarios. The uncertainty set enclosing all 6,000 original in-sample data is represented in Fig. 1(c).

CC-OPF: it is solved through both scenario and robust 
approaches. Two distinct values for the level of confidence $\epsilon$ are used (5\% and 10\%) arising in different numbers of randomly extracted samples (respectively, $N=3,582$ and 1,791 for $\beta=10^{-4}$ ) ensuring the feasibility of the overall set of constraints in $95 \%$ or $90 \%$ of the cases. With the scenario approach, they are treated as equiprobable scenarios, while with the robust one, a box uncertainty set is generated to enclose all of them. Fig. 1(d) shows the randomly extracted samples and the resulting box uncertainty set for $\epsilon=0.05$.

The stochastic, adaptive robust and chance-constrained variants of the OPF problem are implemented and solved under GAMS 24.6.1 using CPLEX 12.6.3. We now describe the out-of-sample results that are presented in Table I. Among all results obtained from the different techniques, those of deterministic OPF are the worst ones in the sense that the resulting system cost and its standard deviation are the highest.

Implementing the DA decisions of the S-OPF yields a lower expected cost compared to the deterministic approach. Indeed, load shedding occurs less often but still takes place in some extreme events, e.g., mean load curtailment is reduced to 0.87 MW instead of 9.32 MW when using 30 scenarios. The expected cost is further decreased when more scenarios are used at the DA stage (i.e., 100 scenarios) since a more accurate characterization of uncertainty is achieved. Adopting a riskaverse attitude results in a higher expected cost compared to the risk-neutral S-OPF but with a significant reduction of load curtailment and thus, a decrease of standard deviation. In this case, the increase of scenarios considered at the DA stage results in a decrease of load shedding (and standard deviation of system cost) since the focus is placed on extreme scenarios.

The DA decisions of the AR-OPF model result in different levels of conservatism depending on the size of uncertainty set. In all cases, the standard deviation of system cost is relatively low, while the expected cost reduces when shrinking the uncertainty set. However, the system is not fully immunized against extreme scenarios in which load curtailment may occur when the uncertainty set is shrunk. Moreover, the definition of the uncertainty set over 30 scenarios allows to further decrease the size of the uncertainty set, while still using relevant data.

The decisions of the chance-constrained OPF based on the scenario approach attain similar results with the S-OPF in terms of expected cost and its standard deviation. However, more scenarios are required to solve the $\mathrm{CC}-\mathrm{OPF}$ models. In the robust reformulation, the uncertainty sets computed to enclose in-sample data are at least as big as the ones used in the AR-OPF, ending up in even more conservative solutions. The reason for that is that the box uncertainty set utilized to enclose the number $N$ of randomly extracted samples leads to over-conservative size of uncertainty sets.

\section{CONCLUSION}

In the present work, we implement stochastic programming, robust optimization and chance-constrained programming in the framework of a two-stage OPF problem with uncertain wind power generation. We make no assumption on the underlying probability distribution and use historical data for
TABLE I: Out-of-sample results

\begin{tabular}{l|l|l|l|l|l}
\hline Method & $\begin{array}{l}\text { Expected } \\
\text { system } \\
\text { cost }[€]\end{array}$ & $\begin{array}{l}\text { Standard } \\
\text { devia- } \\
\text { tion } \\
{[€]}\end{array}$ & $\begin{array}{l}\text { Mean } \\
\text { load cur- } \\
\text { tailment } \\
{[\mathrm{MW}]}\end{array}$ & $\begin{array}{l}\text { Mean } \\
\text { wind } \\
\text { spillage } \\
{[\mathrm{MW}]}\end{array}$ & $\begin{array}{l}\text { Compu- } \\
\text { tation } \\
\text { time } \\
{[\mathrm{s}]}\end{array}$ \\
\hline \multicolumn{6}{c}{ Deterministic OPF } \\
\hline Deterministic & 16,810 & 9,398 & 9.32 & 19.62 & 0.4 \\
\hline \multicolumn{6}{c}{ Stochastic OPF } \\
\hline 30 scenarios & 15,375 & 4,573 & 0.87 & 8.25 & 0.7 \\
100 scenarios & 15,367 & 4,971 & 1.43 & 9.83 & 1.6 \\
\hline \multicolumn{6}{c}{ Risk-averse stochastic OPF $(k=1, \alpha=0.95)$} \\
\hline 30 scenarios & 16,078 & 3,565 & 0.13 & 10.07 & 5.6 \\
100 scenarios & 16,277 & 3,456 & 0.02 & 8.88 & 9.2 \\
\hline \multicolumn{6}{c}{ Adaptive Robust OPF } \\
\hline All 6,000 samples & 16,514 & 3,486 & 0 & 7.21 & 2.6 \\
1,000 random samples & 16,372 & 3,458 & 0.01 & 8.13 & 1.8 \\
100 random samples & 16,318 & 3,450 & 0.01 & 8.53 & 2.1 \\
30 scenarios & 16,293 & 3,453 & 0.02 & 8.74 & 1.9 \\
\hline \multicolumn{7}{c}{ Chance-constrained OPF $\left(\beta=10^{-4}\right)$} \\
\hline Scenario approach $(\epsilon: 0.05)$ & 15,364 & 4,853 & 1.26 & 9.39 & 119.7 \\
Scenario approach $(\epsilon: 0.1)$ & 15,366 & 4,961 & 1.42 & 9.79 & 31.3 \\
Robust approach $(\epsilon: 0.05)$ & 16,545 & 3,493 & 0 & 7.03 & 1.3 \\
Robust approach $(\epsilon: 0.1)$ & 16,546 & 3,493 & 0 & 7.02 & 1.3 \\
\hline
\end{tabular}

the uncertainty characterization. A consistent out-of-sample analysis shows the advantages and disadvantages of each implemented technique. Through our analysis, it can be noticed that conservative approaches result in higher expectation of out-of-sample costs but with a reduced variability.

For repetitive decision processes, stochastic programming is more favorable since it exhibits the lower cost in expectation. However, for a unique long-term decision, the decision maker could prefer to take less risk and opt for robust optimization (e.g., transmission expansion planning). The results of the chance-constrained OPF are attractive under the scenario approach but still too conservative with the robust reformulation.

For further research, individual chance constraints and their approximation through scenario approach or their reformulation under a given distribution shape can be explored. Other uncertainty modeling techniques can also be implemented like distributionally robust optimization, which minimizes the worst-case expected cost among all potential distributions of uncertainty, collected in an ambiguity set.

\section{REFERENCES}

[1] J. M. Morales, A. J. Conejo, H. Madsen, P. Pinson and M. Zugno, "Integrating Renewables in Electricity Markets: Operational Problems," Springer, 2014.

[2] D. Bertsimas, D. B. Brown and C. Caramanis, "Theory and applications of robust optimization," in SIAM Review, vol. 53(3), pp. 464-501, 2011.

[3] D. Bienstock, M. Chertkov and S. Harnett, "Chance-constrained optimal power flow: Risk-aware network control under uncertainty," in SIAM Review, vol. 56(3), pp. $461-495,2014$.

[4] A. Nemirovski, "On safe tractable approximations of chance constraints," in European Journal of Operational Research, vol 219(3), pp. 707-718, 2012.

[5] M. Zugno and A. J. Conejo, "A robust optimization approach to energy and reserve dispatch in electricity markets," in European Journal of Operational Research, vol. 247(2), pp. 659-671, 2015.

[6] Y. Dvorkin, H. Pandžić, M. A. Ortega-Vazquez, D. S. Kirschen, "A hybrid stochastic/interval approach to transmission-constrained unit commitment," in IEEE Transactions on Power Systems, vol. 30(2), pp. 621-631, 2015.

[7] H. Pandžić, Y. Dvorkin, T. Qiu, Y. Wang, and D. S. Kirschen, "Toward costefficient and reliable unit commitment under uncertainty," in IEEE Transactions on Power Systems, vol. 31(2), pp. 970-982, 2016.

[8] R. T. Rockafellar and S. Uryasev, "Optimization of conditional value-at-risk," in Journal of Risk, vol. 2(3), pp. 21-42, 2000.

[9] K. Margellos, P. Goulart and J. Lygeros, "On the road between robust optimization and the scenario approach for chance constrained optimization problems," in IEEE Transactions on Automatic Control, vol. 59(8), pp. 2258-2263, 2014.

[10] A. Arrigo, C. Ordoudis, J. Kazempour, Z. De Grève, J.-F. Toubeau and F. Vallée, "A modified version of the IEEE 24-bus RTS," 2018. [Online]. Available: https: //doi.org/10.5281/zenodo.1493133

[11] A. J. Conejo, M. Carrión, J. M. Morales, "Decision Making Under Uncertainty in Electricity Markets," Springer, 2010. 